\title{
REVIEW
}

\section{Maternal C-reactive protein for detection of chorioamnionitis: An appraisal}

\author{
VIROJ WIWANITKIT \\ Department of Laboratory Medicine, Faculty of Medicine, Chulalongkorn University, Bangkok, Thailand
}

(Received 9 April 2004; accepted 11 October 2004)

\begin{abstract}
Premature delivery is still a significant problem in obstetrics, and chorioamnionitis is an unwelcome complication. C-reactive protein (CRP) is a circulating marker of low-grade inflammation and the role of its measurement in clinical practice remains unclear for many conditions. It has been claimed that estimation of CRP is helpful in the diagnosis of chorioamnionitis, and this study aims to appraise such claims. Following review of the literature, six reports were recruited for further metanalysis, including 466 cases. The overall prevalence of chorioamnionitis was $41 \%(191 / 466)$. The overall diagnostic activity showed sensitivity, specificity, false-positives and false-negatives of $72.8 \%, 76.4 \%, 23.6 \%$ and $27.2 \%$, respectively. Therefore, we can conclude that estimation of maternal CRP is not helpful in the detection of chorioamnionitis, compared with standard investigations.
\end{abstract}

Keywords: Chorioamnionitis, C-reactive protein

\section{Introduction}

Premature delivery is still a significant problem in obstetrics [1]. It has multiple causes; about $50 \%$ of cases are thought to be due to infection. Potential pathogens largely arise from the ascending route and from the endogenous vaginal flora, and may cause chorioamnionitis [1]. The recent increase in knowledge about infection and preterm delivery has engendered many new questions and should make us rethink our long-held beliefs and management strategies [2]. During threatened preterm delivery, general and local infections increase the risks of amniotic infection, premature rupture of membranes (PROM), preterm delivery, and neonatal and/or maternal morbidity of infectious origin [3].

Chorioamnionitis is a totally unwanted complication. Systematic vaginal and blood sampling is helpful in determining the appropriate option in this situation [1-3]. The various serologic and amniotic fluid tests that may identify activation of the host immune and inflammatory responses as a consequence of the microbial invasion of the amniotic cavity are very promising, but not yet fully confirmed for clinical use [1-3]. Examples of these are Creactive protein (CRP), leukocyte count, vaginal microbiological studies and histological study. These tests may identify the early stages of an infectious process, before the full clinical manifestations of chorioamnionitis.

Here, the author appraises the diagnostic properties of maternal CRP for detection of chorioamnionitis. According to this study, this test has only fair sensitivity and specificity.

\section{Materials and methods}

A literature review was performed by means of PubMed (www.pubmed.com) to identify previous reports on the effectiveness of maternal serum CRP measurement compared with standard histological or microbiological methods to detect chorioamnionitis. The operative definition of chorioamnionitis was either histological, which may be asymptomatic, and/ or clinical. The available reports were collected and extracted for the data on the diagnostic properties of

Correspondence: Viroj Wiwanitkit, Department of Laboratory Medicine, Faculty of Medicine, Chulalongkorn University, Bangkok 10330, Thailand.

E-mail: wviroj@yahoo.com

ISSN 1064-7449 print/ISSN 1098-0997 online (C) 2005 Taylor \& Francis

DOI: $10.1080 / 10647440500068321$ 
serum CRP. The primary data were used for metanalysis. Reports that did not present the diagnostic properties (sensitivity, specificity, false positives and false negatives) were excluded. The overall diagnostic properties (sensitivity, specificity, false positives and false negatives) were then calculated. SPSS 11.0 for Windows was used for statistical analysis.

\section{Results}

Six reports [4-9] were recruited for further metanalysis (Tables I and II), including 466 cases. The overall prevalence of chorioamnionitis was $41.0 \%$ (191/466). The overall diagnostic sensitivity, speci- ficity, false positives and false negatives of maternal CRP measurement were $72.8 \%, 76.4 \%, 23.6 \%$ and $27.2 \%$, respectively (Table III).

\section{Discussion}

CRP is a circulating marker of low-grade inflammation, thrombosis and vascular injury, and it has been associated with underlying inflammatory processes $[10,11]$. The role of the measurement of CRP in clinical practice remains unclear for many diseases $[10,11]$, although it has been proposed as helpful in the diagnosis of chorioamnionitis. Among the many previous reports on this topic, some have suggested that CRP has good diagnostic properties $[5,8]$ for the

Table I. Basic demographics of subjects in previous reports on diagnostic properties of maternal serum CRP measurement compared with histological or microbiological investigations in detection of chorioamnionitis.

\begin{tabular}{|c|c|c|c|c|}
\hline Authors & Age group ${ }^{\star}$ & Gestational age ${ }^{\star}$ & Parity* & Race* $^{\star}$ \\
\hline Sereepapong et al., 2001 [4] & Adult & After 28 weeks & Nulliparity, multiparity & Thailand \\
\hline Nowak et al., 1998 [5] & Adult & Before 35 weeks & Nulliparity, multiparity & Poland \\
\hline Steinborn et al., $2000[6]$ & Adult & Before 37 weeks & Nulliparity, multiparity & Germany \\
\hline Mazor et al., 1993 [7] & Adult & Before 37 weeks & Nulliparity, multiparity & Israel \\
\hline Ibarra Chavarria et al., 1989 [8] & Adult & 28 to 35 weeks & Nulliparity, multiparity & Spain \\
\hline Teichmann et al., 1990 [9] & Adult & After 28 weeks & Nulliparity, multiparity & Germany \\
\hline
\end{tabular}

CRP, C-reactive protein; ${ }^{\star}$ these parameters have been shown not to affect the prevalence of disease.

Table II. Details of maternal serum CRP measurement in previous reports on its diagnostic properties compared with histological or microbiological investigations in detection of chorioamnionitis.

\begin{tabular}{lllll}
\hline Author & Indication & Method & Proximity to diagnosis & Confirmation \\
\hline Sereepapong et al., 2001 [4] & PROM & Turbidimetry & Immediate & Histology \\
Nowak et al., 1998 [5] & PROM & ELISA & At least 2 days & Histology \\
Steinborn et al., 2000 [6] & Preterm labor & ELISA & Immediate & Histology \\
Mazor et al., 1993 [7] & Preterm labor & ELISA & Immediate & Microbiology \\
Ibarra Chavarria et al., 1989 [8] & PROM & Nephelometry & Immediate & Histology \\
Teichmann et al., 1990 [9] & AIS & ELISA & Immediate & Histology \\
\hline
\end{tabular}

CRP, C-reactive protein; AIS, amniotic infection syndrome; PROM, premature rupture of membranes; ELISA, enzyme-linked immunosorbent assay; ${ }^{\star}$ these parameters have been shown not to affect the prevalence of disease.

Table III. Sensitivity and specificity in previous reports on diagnostic properties of maternal serum CRP measurement compared with histological or microbiological investigations in detection of chorioamnionitis.

\begin{tabular}{lcccc}
\hline Author & Number of subjects & $\begin{array}{c}\text { Cases of } \\
\text { chorioamnionitis }\end{array}$ & Sensitivity (\%) & Specificity (\%) \\
\hline Sereepapong et al., 2001 [4] & 126 & 50 & 56.0 & 58.0 \\
Nowak et al., 1998 [5] & 80 & 59 & 88.0 & 86.0 \\
Steinborn et al., 2000 [6] & 97 & 48 & 75.5 & 71.4 \\
Mazor et al., 1993 [7] & 48 & 7 & 71.5 & 73.2 \\
Ibarra Chavarria et al., 1989 [8] & 30 & 17 & 94.1 & 100.0 \\
Teichmann et al., 1990 [9] & 85 & 10 & 50.0 & 93.3 \\
\hline
\end{tabular}

CRP, C-reactive protein. 
detection of chorioamnionitis, whereas others have found the opposite $[4,6,7,9]$.

The present study revealed a high prevalence of chorioamnionitis among the study population, which underlines the need for an efficient test for the condition. However, in this respect the overall sensitivity and specificity of maternal CRP measurement were only fair, with high false-positive and false-negative rates. We therefore conclude that maternal CRP measurement is not a good tool for the detection of chorioamnionitis, compared with standard tests.

\section{References}

1. Garland SM, Ni Chuileannain F, Satzke C, Robins-Browne R. Mechanisms, organisms and markers of infection in pregnancy. J Reprod Immunol 2002;57:169-183.

2. Asrat $T$. Intra-amniotic infection in patients with preterm prelabor rupture of membranes. Pathophysiology, detection, and management. Clin Perinatol 2001;28:735-751.

3. Subtil D. Prognostic and therapeutic value of biologic signs of infection in the management of preterm labor (amniocentesis excepted). J Gynecol Obstet Biol Reprod (Paris) 2002; 31(7):5S43-51

4. Sereepapong W, Limpongsanurak S, Triratanachat S, Wannakrairot P, Charuruks N, Krailadsiri P. The role of maternal serum C-reactive protein and white blood cell count in the prediction of chorioamnionitis in women with premature rupture of membranes. J Med Assoc Thai 2001;84(1):S360 S366.
5. Nowak M, Oszukowski P, Szpakowski M, Malinowski A, Maciolek-Blewniewska G. Intrauterine infections. I. The role of C-reactive protein, white blood cell count and erythrocyte sedimentation rate in pregnant women in the detection of intrauterine infection after preliminary rupture of membranes. Ginekol Pol 1998;69:615-622.

6. Steinborn A, Sohn C, Scharf A, Geka F, Heger S, Kaufmann M. Serum intercellular adhesion molecule-1 levels and histologic chorioamnionitis. Obstet Gynecol 2000;95:671676.

7. Mazor M, Kassis A, Horowitz S, Wiznitzer A, Kuperman O, Meril C, Glezerman M. Relationship between C-reactive protein levels and intraamniotic infection in women with preterm labor. J Reprod Med 1993;38:799-803.

8. Ibarra Chavarria V, Sanhueza Smith P, Mota Gonzalez M, del Rey Pineda G, Karchmer S. C-reactive protein as early marker of chorioamnionitis in premature rupture of membranes. Ginecol Obstet Mex 1989;57:203-208.

9. Teichmann AT, Arendt P, Speer CP. Premature rupture of the membranes and amniotic infections - the significance of laboratory tests. Eur J Obstet Gynecol Reprod Biol 1990;34:217-222.

10. Theuma P, Fonseca VA. Inflammation and emerging risk factors in diabetes mellitus and atherosclerosis. Curr Diab Rep 2003;3:248-254.

11. Pepys MB, Hirschfield GM. C-reactive protein: a critical update. J Clin Invest $2003 ; 111: 1805-1812$.

12. Bek KM, Nielsen FR, Qvist I, Rasmussen PE, Tobiassen M. C-reactive protein (CRP) and pregnancy. An early indicator of chorioamnionitis. A review. Eur J Obstet Gynecol Reprod Biol 1990;35:29-33. 


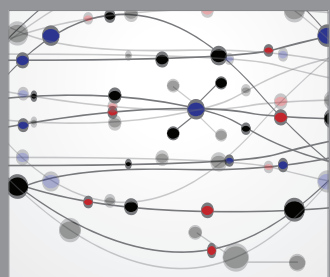

The Scientific World Journal
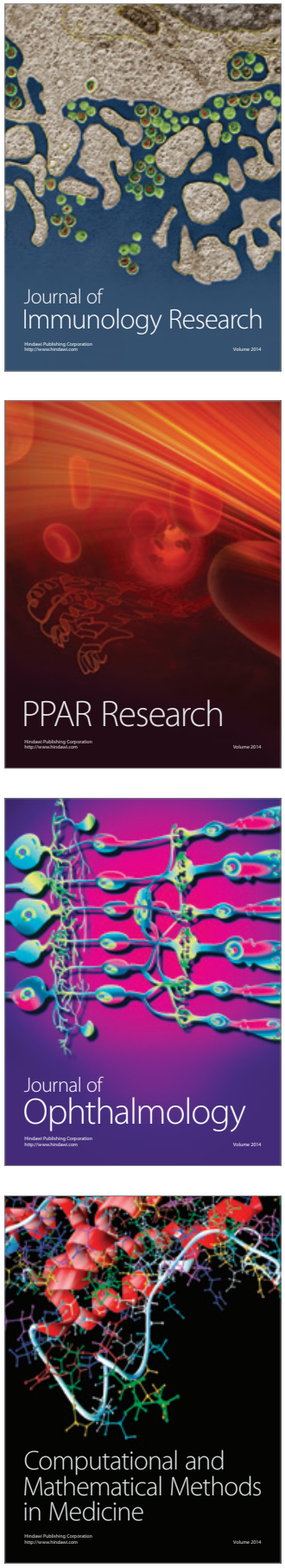

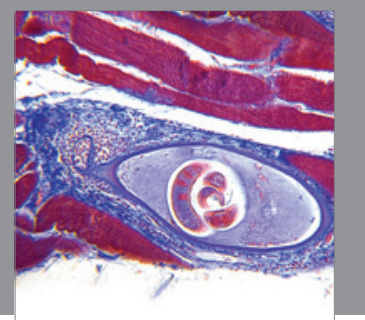

Gastroenterology

Research and Practice
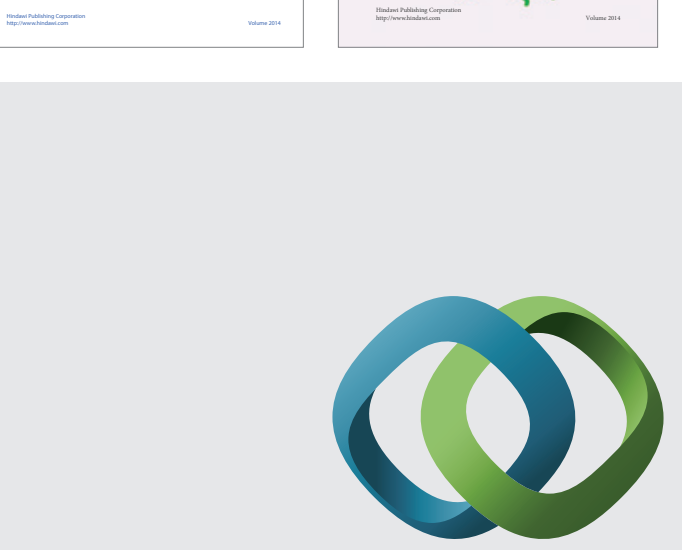

\section{Hindawi}

Submit your manuscripts at

http://www.hindawi.com
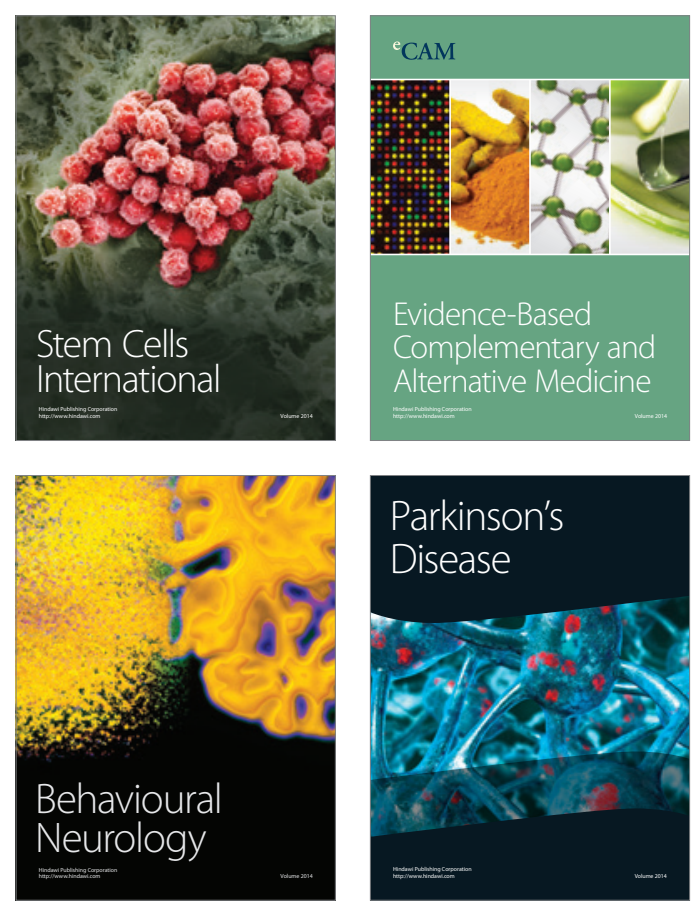

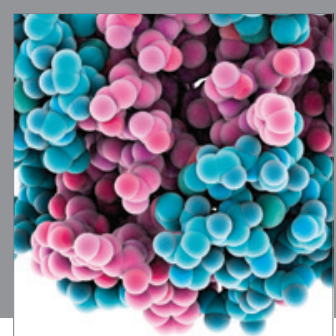

Journal of
Diabetes Research

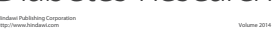

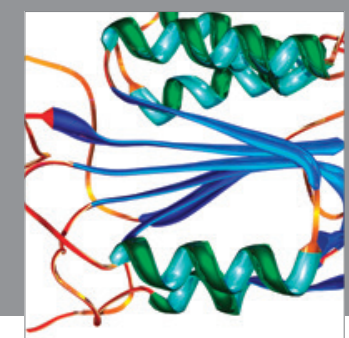

Disease Markers
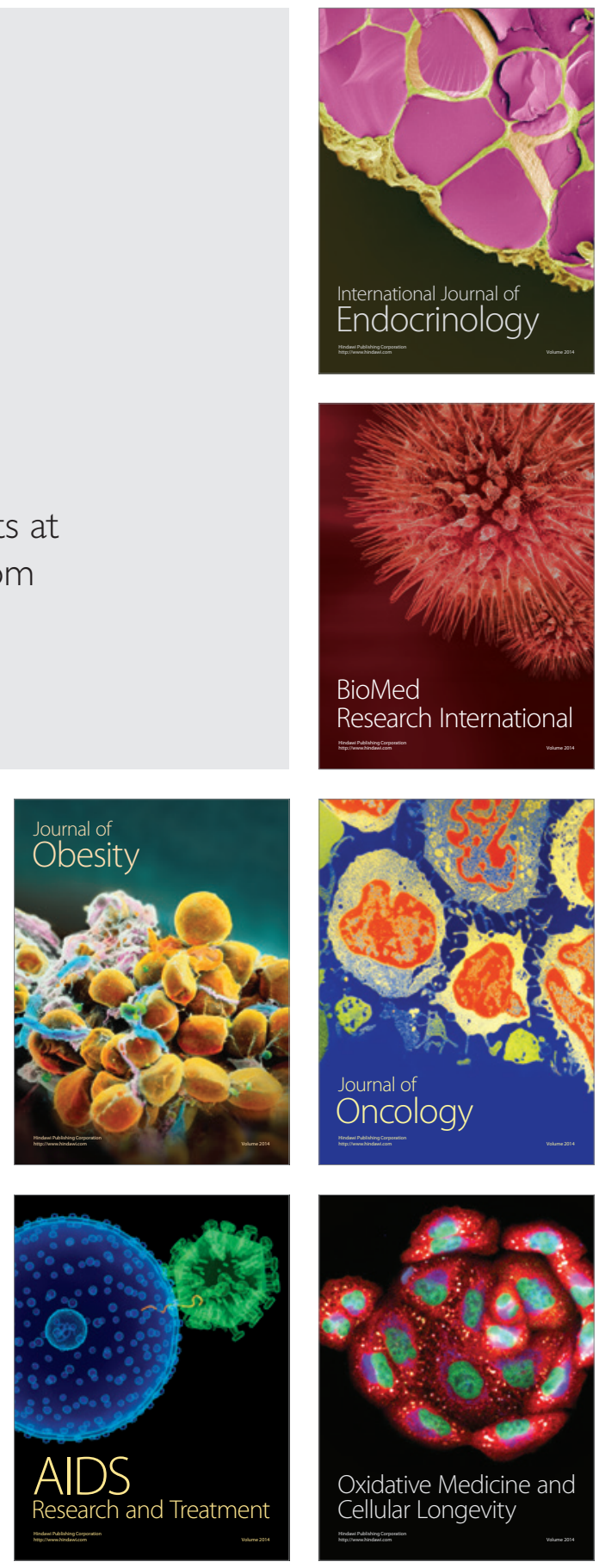